\title{
Reviewer Acknowledgment
}

The Editors of Genetics in Medicine would like to thank all of the individuals who have provided reviews of manuscripts over the past year. These reviews have been critical to our mission, enhancing the knowledge and practice of medical genetics and allowing the publication of high-quality papers in Genetics in Medicine.

Louise Acheson, MD, MS

Mary Ahrens, MS

Raye Lynn Alford, PhD

Jean Amos, $\mathrm{PhD}$

Alexander Asamoah, MD, PhD

David Asch, PhD

Michael Barry, PhD

James Barton, MD

Arthur Beaudet, MD

Laura Beskow, MPH

Frederick Bieber, $\mathrm{PhD}$

Barbara Biesecker, MS

Amanda Brown, PhD

Charlotte Brown, $\mathrm{PhD}$

John Brunzell, MD

Peter Byers, MD

John Carey, MD

Stephen Cederbaum, MD

Ellen Clayton, MD, JD

Mario Cleves, $\mathrm{PhD}$

Alberto Cornier, MD

Adolpho Correa, MD, PhD

Cynthia Curry, MD

Mary Curtis, MD

Gary Cutting, MD

Thomas Daly, MD

Katrina Dipple, MD, PhD

Luba Djurdjinovic, MS

James Eckman, MD

Charles Epstein, MD

Richard Erbe, MD

Deborah Eunpu, MS

Jane Evans, PhD

Carolyn Farrell, MS, CNP

Claude Ferec, MD, PhD

Nancy Fisher, MD, MPH

David Flannery, MD

Norman Fost, MD

Michelle Fox, MS

Christopher Friedrich, MD, PhD

Phillipe Froguel, MD, PhD

Pat Gaffney, MD

Montserrat Garcia Closas, MD, DrPH

Thomas Gelehrter, MD

Jill Goldman, MS, MPH
Gregory Grabowski, MD

Wayne Grody, MD, PhD

Marta Gwinn, MD, MPH

James Haddow, MD

Ada Hamosh, MD

James Hanson, MD

Robert Hegele, MD

Gail Herman, MD, PhD

J. Greg Howe, $\mathrm{PhD}$

Jamie Israel, MS

Ramaswamy Iyer, $\mathrm{PhD}$

Ethylin Wang Jabs, MD

Colleen Jackson-Cook, $\mathrm{PhD}$

Marilyn Jones, MD

Feige Kaplan, $\mathrm{PhD}$

Mohamed Khalifa, MD

William Kimberling, $\mathrm{PhD}$

David Kwiatkowski, MD, PhD

Ronald Lacro, MD

Allen Lamb, PhD

Janine LaSalle, $\mathrm{PhD}$

David Ledbetter, PhD

Nicholas Lench, BSc, PhD

Bonnie LeRoy, MS

Howard Levy, MD, PhD

Raymond Lewandowski, MD

Julian Little, $\mathrm{PhD}$

Kim Martin, MD

Anna Mastroianni, JD, MPH

Reuben Matalon, MD, PhD

Anne Matthews, $\mathrm{PhD}$

Edward R. B. McCabe, MD, PhD

Linda McCabe, $\mathrm{PhD}$

Deborah McDermott, MS

Margaret McGovern, PhD, MD

Scott McIvor, $\mathrm{PhD}$

Jeff Milunsky, MD

John Mulvihill, MD

Kasinathan Muralidharan, $\mathrm{PhD}$

Walter Nance, MD, PhD

Katherine Nathanson, MD

Hope Northrup, MD

Thomas O'Brien, $\mathrm{PhD}$

William Oetting, $\mathrm{PhD}$

Susan Olson, $\mathrm{PhD}$
Vivian Ota Wang, $\mathrm{PhD}$

Susan Panny, MD

Gregory Pastores, MD

Sharon Plon, MD, PhD

Bradley Popovich, MD, MSc

Lorraine Potocki, MD

Thomas Prior, $\mathrm{PhD}$

Reed Pyeritz, MD, PhD

Laura Ranum, $\mathrm{PhD}$

Philip Reilly, MD, JD

C. Sue Richards, PhD

Benjamin Roa, PhD

Nathaniel Robin, MD

Patricia Roche, JD, M.Ed

Wendy Rubenstein, MD, PhD

Howard Saal, MD

Lauren Scheuer, MD

Maren Scheuner, MD, MPH

Katherine Schneider, MPH

Debra Schutte, PhD, RN

Joe Leigh Simpson, MD

Richard J. H. Smith, MD

Moyra Smith, MD, PhD

Michael Stehney, MD, MPH

Gail Stetten, PhD

Eva Sujansky, MD

James Sutcliffe, $\mathrm{PhD}$

Sapna Syngal, MD, MPH

James Tepperberg PhD

George Thomas, $\mathrm{PhD}$

Helga Toriello, PhD

William Toscano, $\mathrm{PhD}$

Daniel Van Dyke, PhD

Brian Weinshenker, MD

Richard Wenstrup, MD

Lari Wenzel, $\mathrm{PhD}$

Judith Westerman, MD

Chet Whitley, PhD, MD

Georgia Wiesner, MD, MS

David Witt, MD

John Witte, PhD

Bai-Lin $\mathrm{Wu}, \mathrm{PhD}$

Paula Yoon, ScD, MPH 